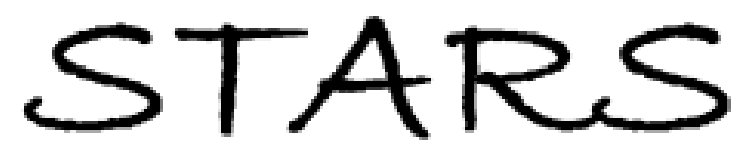

University of Central Florida

STARS

Faculty Bibliography 2000s

Faculty Bibliography

$1-1-2004$

\title{
Tunable-focus flat liquid crystal spherical lens
}

\author{
Hongwen Ren \\ University of Central Florida \\ Yun-Hsing Fan \\ University of Central Florida \\ Sebastian Gauza \\ University of Central Florida \\ Shin-Tson Wu \\ University of Central Florida
}

Find similar works at: https://stars.library.ucf.edu/facultybib2000

University of Central Florida Libraries http://library.ucf.edu

This Article is brought to you for free and open access by the Faculty Bibliography at STARS. It has been accepted for inclusion in Faculty Bibliography 2000 s by an authorized administrator of STARS. For more information, please contactSTARS@ucf.edu.

\section{Recommended Citation}

Ren, Hongwen; Fan, Yun-Hsing; Gauza, Sebastian; and Wu, Shin-Tson, "Tunable-focus flat liquid crystal spherical lens" (2004). Faculty Bibliography 2000s. 4734.

https://stars.library.ucf.edu/facultybib2000/4734

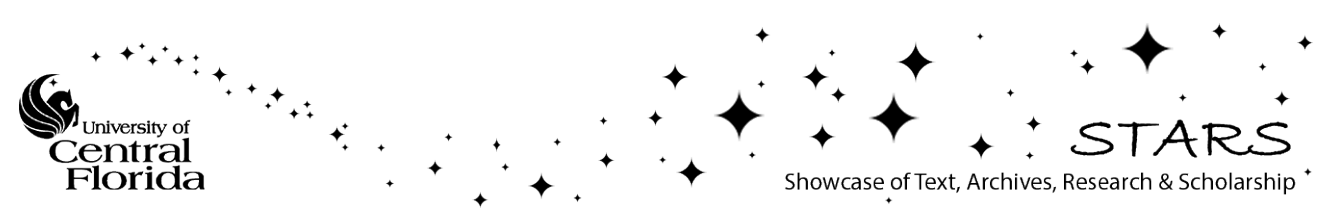




\title{
Tunable-focus flat liquid crystal spherical lens
}

\author{
Hongwen Ren, Yun-Hsing Fan, Sebastian Gauza, and Shin-Tson Wu ${ }^{\text {a) }}$ \\ School of Optics, University of Central Florida, Orlando, Florida 32816
}

(Received 26 February 2004; accepted 16 April 2004; published online 21 May 2004)

\begin{abstract}
A tunable-focus spherical lens using two flat substrates and inhomogeneous electric field over a homogeneous liquid crystal (LC) layer is demonstrated. The top flat substrate has an imbedded spherical indium-tin-oxide (ITO) electrode and the bottom has a planar ITO electrode on its inner surface. The inhomogeneous electric field generates a centrosymmetric gradient refractive index profile within the LC layer which causes the focusing behavior. The focal length of the LC lens can be tuned continuously from infinity to $0.6 \mathrm{~m}$ by the applied voltage. (C) 2004 American Institute of Physics. [DOI: $10.1063 / 1.1760226]$
\end{abstract}

Since liquid crystal (LC) was proposed for making a tunable lens, ${ }^{1}$ the LC based zoom lens has been studied extensively for machine vision, photonics, information processing, and eye glasses. Various attempts such as surface relief profile, ${ }^{2-4}$ line or hole-patterned electrode, ${ }^{5-8}$ Fresnel zone type, ${ }^{9,10}$ modal control, ${ }^{11}$ as well as polymer network LC technique ${ }^{12,13}$ have been demonstrated. Among these approaches, the lens with surface relief profile which combines a passive solid-state lens and a LC modulator possesses several merits, e.g., simple fabrication, single electrode, and easy to realize a spherical phase profile within the LC layer. However, the LC lens with curved inner surfaces would scatter light due to the poor molecular alignment. ${ }^{2}$ Moreover, its focus tunable range is rather limited. ${ }^{3}$

In this letter, we demonstrated a flat LC lens exhibiting a wide-range tunable focal length. Unlike the surface relief LC lens, the present lens has planar substrates and a uniform LC layer. One of the flat substrates has an imbedded spherical electrode and the other has a planar electrode. The electric field from the spherical and planar electrodes induces a centrosymmetric gradient refractive index distribution within the LC layer which, in turn, causes the focusing effect. The focal length is tunable by the applied voltage. Unlike the nonuniform LC layer approach, our lens exhibits a uniform optical response across the lens aperture due to the homogeneous cell gap. No light scattering or diffraction occurs due to the homogeneous LC alignment and continuous electrode. Both positive and negative lenses can be realized by simply reversing the shape of the spherical electrode.

Figure 1 illustrates the fabrication procedures for a positive LC lens. The concave surface of the bottom glass substrate is coated with a transparent indium-tin-oxide (ITO) electrode, as shown in Fig. 1(a). Next, the sag area could be matched by a convex glass lens with the same curvature or filled with a polymer having the same refractive index as the employed glass substrate to form a planar substrate, as shown in Fig. 1(b). For the demonstration purpose, we filled the sag area with an UV curable prepolymer. To simplify the fabrication process, we used an empty LC cell to seal the prepolymer. The glass substrate which is in contact with the prepolymer has $d_{2}=0.55 \mathrm{~mm}$, but no ITO electrode. When

${ }^{\text {a)} E l e c t r o n i c ~ m a i l: ~ s w u @ m a i l . u c f . e d u ~}$ the prepolymer was cured by UV, the lens and the LC cell were attached together. The inner surfaces of the LC cell are coated with polyimide alignment layers and rubbed in antiparallel direction. The pretilt angle is $\sim 3^{\circ}$. When a LC mixture is injected into the cell, homogeneous alignment is induced by the buffed polyimide layers, as shown in Fig. 1(c).

Based on the above-mentioned procedures, we fabricated a positive lens LC cell. The concave glass lens with radius $R=-9.30 \mathrm{~mm}$, aperture $D=6 \mathrm{~mm}$, and sag $d_{1}=0.34$ $\mathrm{mm}$ was purchased from Edmund Industrial Optics (BK7 glass, $\left.n_{g}=1.517\right)$. The refractive index of the filled polymer $\operatorname{NOA65}\left(n_{p} \sim 1.524\right.$, Norland Optical Adhesive) would affect the initial focal length of the LC lens. If $n_{p} \approx n_{g}$, then the LC device would not focus light in the voltage-off state. If $n_{p}$ is much smaller than $n_{g}$, then the device would have an initial focus. A homemade high birefringence LC mixture designated as UCF-2 $(\Delta n=0.4 \text { at } \lambda=633 \mathrm{~nm})^{14}$ was used to fill the 40- $\mu \mathrm{m}$-cell gap.

To evaluate the optical properties of the spherical lens, we investigated the profile of the phase retardation by observing the interference fringes between the ordinary and extraordinary rays using a white light under crossed polarizers. The rubbing direction of the lens cell is oriented at $45^{\circ}$ with respect to the fast axis of the linear polarizer. Two images of the lens cell at $V=0$ and $V=25 V_{\text {rms }}$ were taken using a digital camera, and results are shown in Figs. 2(a) and 2(b), respectively.

At $V=0$, the sample is optically homogeneous and no interference rings are observed, as shown in Fig. 2(a). This means that the gradient refractive index distribution does not occur in the LC layer. When a voltage is applied to the lens cell, concentric circular rings appear. Figure 2(b) shows the image taken at $V=25 V_{\text {rms }}$. As the applied voltage is increased, the circular rings start to occur from the borders and then gradually expand to the center. This implies that a gradient refractive index is induced in the LC layer and is tunable by the applied voltage. The movement direction of the rings towards the center indicates that the LC in the central area experiences a weaker electric field than that in the borders. This phenomenon basically correlates to the spherical electrode gap. At a given voltage, the electric field in the center of the LC lens is weaker than that in the edges. Further increasing the applied voltage, the numbers of the circu- 

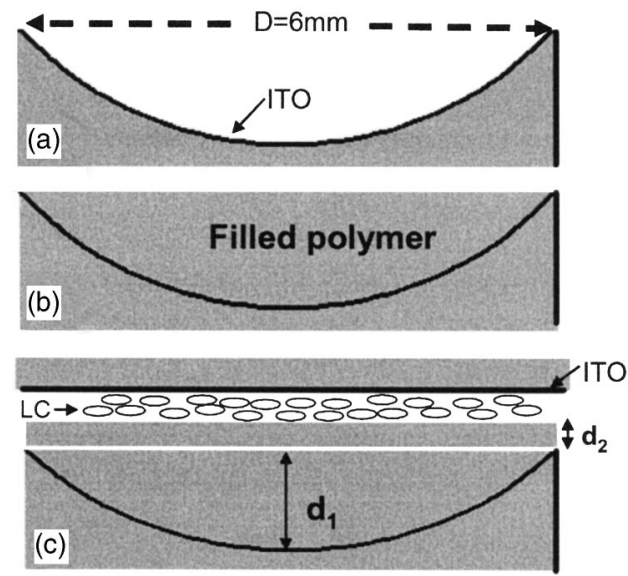

FIG. 1. Procedures for fabricating the spherical LC lens: (a) deposit ITO on a concave glass lens, (b) fill the sag area with polymer, and (c) assemble the LC lens cell with another flat glass substrate.

lar rings increase sharply and then decrease gradually in the high voltage regime where most of the LC directors are reoriented by the electric field.

To characterize the light focusing properties of the lens cell, we measured the $3 \mathrm{D}$ profiles of the outgoing beams (He-Ne laser, $\lambda=633 \mathrm{~nm}$ ) using a CCD camera. The CCD camera was set at $\sim 80 \mathrm{~cm}$ behind the LC lens cell. The intensity profiles were measured at $V=0,23$ and $35 V_{\text {rms }}$, and results are shown in Fig. 3. At $V=0$, the observed $\mathrm{He}-\mathrm{Ne}$ laser beam is not very uniform due to the Gaussian nature of the input laser beam. The peak intensity is $\sim 6$ $\times 10^{3}$ arbitrary units. As the voltage increases to $23 V_{\text {rms }}$, the focusing effect manifests. The measured intensity at the CCD focal plane exceeds $6.5 \times 10^{4}$ arbitrary units. As the voltage is further increased, the peak intensity of the outgoing beam tends to decrease. At $V=35 V_{\text {rms }}$, the peak intensity drops to $1.7 \times 10^{4}$ arbitrary units. This is because the LC molecules in the bulk are reoriented by the electric field. The curvature of the refractive index profile is gradually flattened. As a result, the focal length of the lens increases and the measured light intensity at the CCD focal plane decreases.

To evaluate the image quality of the LC lens, we typed a few lines of "LENS" on a computer screen as an object and the lens was set at $\sim 15 \mathrm{~cm}$ in front of the computer screen. Since the LC lens works for a linearly polarized light, a sheet linear polarizer was placed between the sample and the screen. The rubbing direction of the $\mathrm{LC}$ cell was adjusted to

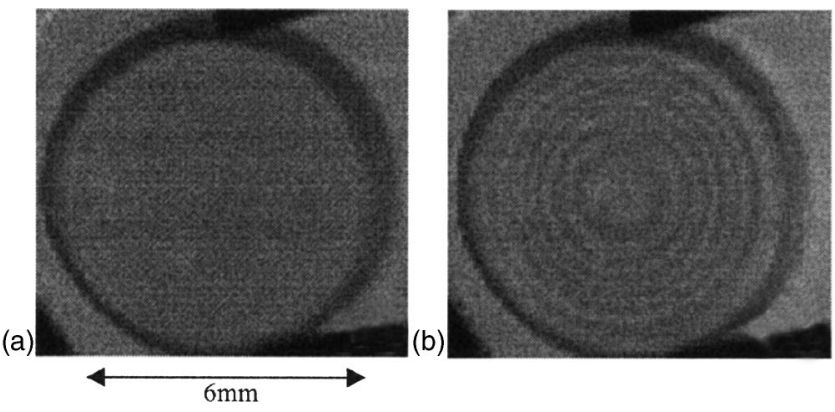

FIG. 2. Interferograms of the circular LC lens at two different operating voltages: (a) $V=0$, (b) $V=25 V_{\text {rms }}$. The lens aperture $D=6 \mathrm{~mm}$. The polarizers are crossed. The rubbing direction of the cell is oriented at $45^{\circ}$ with respect to the fast axis of the linear polarizer. LC used is UCF-2 and cell gap $d=40 \mu \mathrm{m}$.
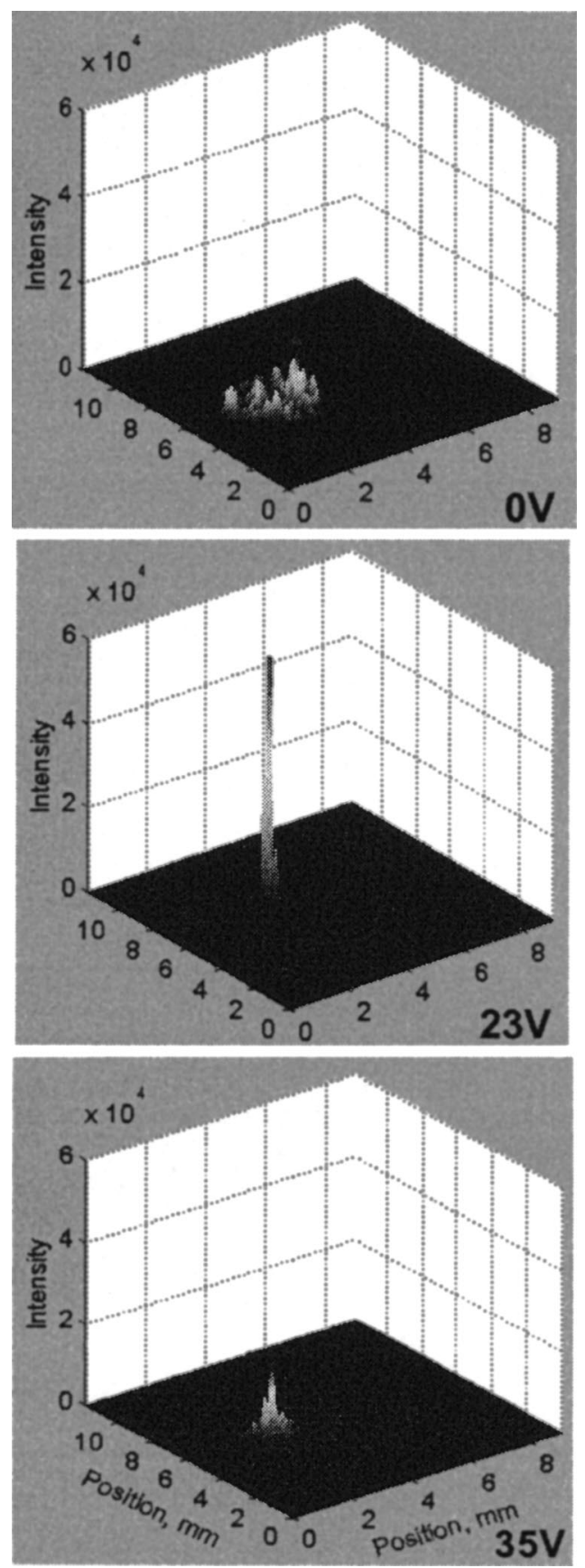

FIG. 3. CCD images of the measured $\mathrm{He}-\mathrm{Ne}$ laser beam intensity profile at $V=0,23$, and $35 V_{\text {rms }}$, respectively.

be parallel to the polarizer's transmission axis. A digital camera was fixed right behind the sample. Two images were taken at $V=0$ and $V=30 V_{\text {rms }}$, as shown in Figs. 4(a) and 4(b), respectively. At $V=0$, a clear image of the typed words is observed. As the applied voltage increases, the observed image is magnified and slightly blurred. The blurred image is mainly due to the defocusing effect. As the applied voltage exceeds $40 V_{\text {rms }}$, the image size is gradually reduced. The larger image means the focal length of the LC lens is longer than the distance between the sample and the object. The magnified image that we observed is actually a virtual image.

The voltage-dependent focal length of the lens was investigated and results are plotted in Fig. 5. At $V=0$, LC directors are aligned homogeneously due to the surface anchoring effect from the substrates. Thus, no focusing effect 

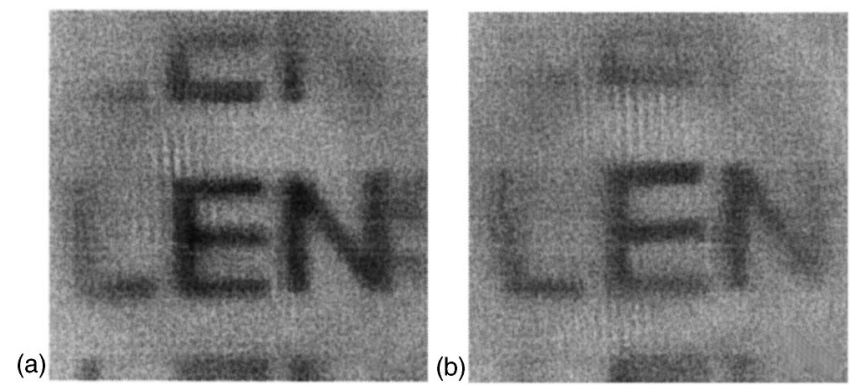

FIG. 4. Imaging behavior of the LC lens at (a) $V=0$ and (b) $V=30 V_{\text {rms }}$.

occurs or the focal point is at infinity. As the voltage increases, the focal length is reduced accordingly. At $V$ $\sim 40 V_{\text {rms }}$, the focal length reaches a minimum ( $f$ $\sim 0.6 \mathrm{~m}$ ). Further increasing the voltage would cause the focal length to bounce back, but at a different rate.

The focal length of an LC lens can be evaluated using the Fresnel's approximation:

$$
f=\frac{r^{2}}{2 \delta n d_{\mathrm{LC}}},
$$

where $r=D / 2$ ( $D$ is the lens aperture), $d_{\mathrm{LC}}$ is the LC layer thickness, and $\delta n$ is the refractive index difference between the lens center and border. From Fig. 1, $\delta n$ is determined by the electric field difference between the lens center and the border. When a voltage $V$ is applied to the lens cell, the electric field in the center $\left(E_{\text {center }}\right)$ and at the border $\left(E_{\text {border }}\right)$ is expressed as:

$$
\begin{aligned}
& E_{\text {center }}=\frac{V / \varepsilon_{\mathrm{LC}}}{\frac{d_{\mathrm{LC}}}{\varepsilon_{\mathrm{LC}}}+\frac{d_{2}}{\varepsilon_{2}}+\frac{d_{1}}{\varepsilon_{1}},} \\
& E_{\text {border }}=\frac{V / \varepsilon_{\mathrm{LC}}}{\frac{d_{\mathrm{LC}}}{\varepsilon_{\mathrm{LC}}}+\frac{d_{2}}{\varepsilon_{2}},}
\end{aligned}
$$

where $\varepsilon_{\mathrm{LC}}, \varepsilon_{2}$, and $\varepsilon_{1}$ represent the dielectric constant of the LC, medium 2 and 1, respectively. In an ideal case, we would like to eliminate the glass substrate which is closer to the spherical electrode, i.e., $d_{2} \sim 0$. In such a condition, the electric field shielding effect due to the glass substrate is reduced and the required operating voltage is lowered. From Eq. (1), the shortest focal length occurs when $\delta n=\Delta n$, i.e., the LC molecules in the border are completely aligned by the electric field while those in the center are not yet reoriented owing to the weaker electric field.

In comparison with other tunable lens technologies, the major advantages of our lens are in simple fabrication pro-

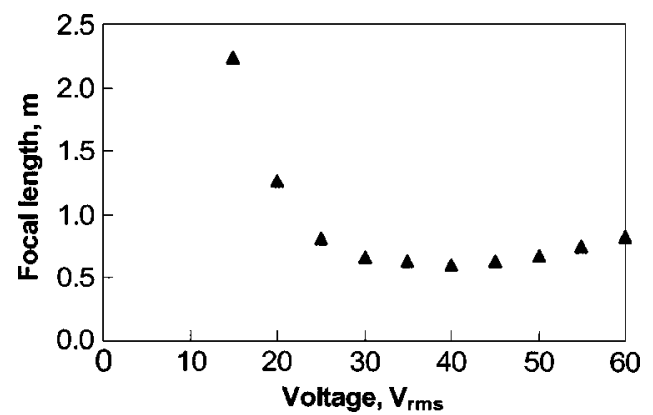

FIG. 5. Voltage-dependent focal length of the LC lens. Lens aperture $D=6$ $\mathrm{mm}$, LC: UCF-2, cell gap $d=40 \mu \mathrm{m}$ and $\lambda=633 \mathrm{~nm}$.

cess, uniform LC cell gap, plano-substrate surface, and simple electrodes. In addition, the lens has a very wide range of tunable focal length without light diffraction and scattering. In theory, the light throughput can reach $100 \%$ for a linearly polarized laser beam. The response time of the lens depends on the LC cell gap. In our experiment, we used a high $\Delta n$ LC mixture and $40 \mu \mathrm{m}$ cell gap. The switching speed is $\sim 1 \mathrm{~s}$ at room temperature.

In conclusion, we have demonstrated an electrically tunable-focus LC lens using the combination of spherical and planar electrodes. Without voltage, the focal length of the LC lens is at infinity due to the flat substrates and homogeneous LC alignment. As the applied voltage increases, the focal length is gradually drawn near from infinity to $\sim 0.6 \mathrm{~m}$. By reversing the shape of the spherical electrode, a negative lens or lens arrays can be easily fabricated.

This work is supported by DARPA BOSS Program under Contract No. DAAD 19-02-1-0208.

${ }^{1}$ C. Bricot, M. Hareng, and E. Spitz, U.S. Patent No. 4,037,929 (1977).

${ }^{2}$ S. Sato, Jpn. J. Appl. Phys., Part 1 18, 1679 (1979).

${ }^{3}$ B. Wang, M. Ye, M. Honma, T. Nose, and S. Sato, Jpn. J. Appl. Phys., Part 2 41, L1232 (2002).

${ }^{4}$ H. S. Ji, J. H. Kim, and S. Kumar, Opt. Lett. 28, 1147 (2003).

${ }^{5}$ T. Nose and S. Sato, Liq. Cryst. 5, 1425 (1989).

${ }^{6}$ M. Ye and S. Sato, Jpn. J. Appl. Phys., Part 2 41, L571 (2002).

${ }^{7}$ N. A. Riza and M. C. DeJule, Opt. Lett. 19, 1013 (1994).

${ }^{8}$ W. W. Chan and S. T. Kowel, Appl. Opt. 36, 8958 (1997)

${ }^{9}$ J. S. Patel and K. Rastani, Opt. Lett. 16, 532 (1991).

${ }^{10}$ H. Ren, Y. H. Fan, and S. T. Wu, Appl. Phys. Lett. 83, 1515 (2003).

${ }^{11}$ A. F. Naumov, M. Yu. Loktev, I. R. Guralnik, and G. Vdovin, Opt. Lett. 23, 992 (1998).

${ }^{12}$ H. Ren and S. T. Wu, Appl. Phys. Lett. 82, 22 (2003).

${ }^{13}$ V. V. Presnyakov, K. E. Asatryan, and T. V. Galstian, Opt. Express 10, 865 (2002).

${ }^{14}$ S. Gauza, H. Wang, C. H. Wen, S. T. Wu, A. J. Seed, and R. Dabrowski, Jpn. J. Appl. Phys., Part 1 42, 3463 (2003). 